\title{
Utilization of Community-based Health Information Systems in Decision Making and Health Action in Nyalenda, Kisumu County, Kenya
}

\author{
Nzanzu Jeremie ", Dan Kaseje, Rose Olayo, Caroline Akinyi \\ Great Lakes University of Kisumu, Kenya \\ *Corresponding Author: jeremienzanzu@gmail.com
}

Copyright @ 2014 Horizon Research Publishing All rights reserved.

\begin{abstract}
Information should form the backbone of decisions that effect change in all areas. It is therefore paramount in health, for information to be used for decision making. In effect this will bring about equity in health resource allocation. The development of comprehensive community based health information systems is increasingly becoming important for measuring and improving the quality of health services. Many developing countries including Kenya have made efforts to strengthen their national health information systems to provide information for decision-making in managing health care services. The purpose of this paper is to explore how data collected at the community level is utilised by various stakeholders within the community in order to produce actionable information for decision making. This was a descriptive, cross sectional study. The qualitative aspects of data collection involved key informant interviews and focused group discussions with the health service providers; potential health services users, and community health workers. Findings indicated that strong local ownership of data developed at the community. The main perspectives of service providers included joint ownership of health intervention programs at the community level, the community owned their health and aimed to improve it and community health workers to champion on health and development issues to bring about change.
\end{abstract}

Keywords Utilization of Data, Community Health Strategy, Community Based Information Systems, Access to Care

\section{Introduction}

A Community based health Information System (CBHIS) is a type of health information system that is based in the rural community and informal settlements of urban areas. Its objective is to produce relevant and quality information to support decision making. The CBHIS should hence strive to complement the functions of a health system which include:
Improving decision-making, improving service delivery to the population and subsequently Impacting positively on the health outcomes of the target population at different levels of the health system. Health systems performance would be enhanced not only by bridging the interface between the community and the health system but also encouraging timely decision making and action based on available information ${ }^{2}$.

A Community Based Information System provides evidence grounded in the community, gathered, analyzed and used by the community and therefore has the potential to improve timely decision making planning and action as well as monitoring of improvement ${ }^{1}$.CBHIS enables community to follow up progress of planned activities towards set objectives thereby ascertaining accountability. Usefulness of CBHIS is greatly enhanced when linked to Health Facility Information System, and used for dialogue, for timely evidence-based decision making. Researchers have demonstrated that respectful dialogue with communities as equal partners improves confidence, as well as the demand and supply sides of the health care system ${ }^{1-2}$.

Community based Health Information System allows health managers and service providers to document, analyze, and use information to improve coverage, continuity, and quality of health care services at all levels by better planning, monitoring, and evaluation of health services ${ }^{2}$

Information systems are increasingly important for measuring and improving the quality and coverage of health services. There is a global shift from curative to preventive care, from hospital care to community and public health care, from centralized to decentralized health care, from a specific project approach to a comprehensive sectoral approach ${ }^{3}$.

The restructuring of health information systems (HIS) has become an important trend in the entire developing world since the adoption of primary health care as a global strategy for achieving 'health for all' goal ${ }^{3}$.

Information is not an end in itself, but a means to better decision making in policy design, health planning, management, monitoring and evaluation of health programs and services including patient care. It improves overall 
health service performance and outcomes ${ }^{4}$. Health management information systems can be defined as a programme that was designed to provide reliable and comprehensive information about the health system to health managers, to enable them to make decisions that would improve the quality of services. It is therefore very important to ensure that there is quality data to be used in decision making. Such decisions would be valid and reliable because they would be evidence based ${ }^{3-4}$.

This study seeks to find out how locally generated data is used for decision making and health action by the community and health service providers in Nyalenda.

\section{Literature Review}

It is increasingly imperative for health managers and service providers to collect, analyzed and use data from the field for planning health interventions in the community that need it most ${ }^{2}$. One of the building blocks of health systems is a well-functioning health information system that ensures the production, analysis, dissemination and use of reliable and timely information on health determinants, health system performance and health status ${ }^{3}$. The essence of health information is seen in the implementation of programmes meant to improve care in the community; this is so in that for an evidence-based management to succeed, the need for an organized information is paramount; an organized information does not emanate from a vacuum, it is people who collect them and place them in a format that is accessible, understandable and easy to interpret and use.

It is thus of great importance that health service providers are trained in information gathering and analytical techniques to allow them to be able to use available information for the right purpose, and this is the best way to improve health governance at community $\operatorname{level}^{2-3}$; it also allow for efficient use of scarce health resources for maximum benefit to the people who need it most at the grass-root level.

The generation and the strategic use of information, intelligence and research on health and health systems is an integral part of leadership and governance function ${ }^{5}$, which should support health information system to be able to generate population and facility based data censuses, household surveys, registration data, public health surveillance, medical records, data on health services and health system resources etc.; it must also have the capacity to detect, investigate, communicate and contain events that threaten public health security at the place they occur and as soon as they occur; finally it must have the capacity to synthesize information and promote the availability and application of this knowledge ${ }^{6}$.

WHO supports the development of strong public health surveillance systems, as part of an inter-connected global system to collectively reduce international vulnerability to public health threats; thus under health information system, the priority for systematic improvement are in the areas of national information systems, reporting, stronger national surveillance and response capacity, tracking performance, 'standards, methods and tools', as well as synthesis and analysis of country, regional and global data ${ }^{3-8}$

\section{Health information system in Kenya}

Ramana et al, observed that health management information system remains the source of information for the Annual Operation plan for the Ministry of Health in Kenya; however like most developing countries, Chaulagai, (2005), there are challenges in getting reliable information in time from the health management information system; the major challenges include inconsistencies in data reported compared to actual sources of data, weak capacities of health management information staff, lack of integration among the many parallel data collection systems and inadequate coordination. These challenges point to one critical missing link that Odhiambo-Otieno et al, (2005) observed as the lack of an evaluation criteria for established health information management system to allow for organized and accurate information gathering by the health workers.

The premise of this study is the Kenya Essential Package for Health, for which the community health strategy was established as a vehicle for widening access to essential health care at the grass-root ${ }^{8}$. As a new concept the success of the community health strategy, depended on the establishment of evidence-based dialogue informed by community and facility based information systems; this allow for the health service providers to make decisions on health interventions from an informed perspective ${ }^{7}$. Setting up the health information system entails analyzing information collected by the community health workers and supporting task groups, facilitating regular evidence-based dialogue and community days as well as disseminating analyzed information for dialogue, advocacy and social mobilization. Understanding of the role played by the health managers in using health information is crucial in assessing health intervention outcomes; WHO posits that understanding the dominant and conflicting logics at play can provide implementers with deep insight into aspects to examine when performing needs and situational analyses as well as when developing implementation and change management strategies.

\section{Operation of the community based health information system in Kenya}

The community based health information system is meant to generate health-related information through sources at the community level with the potential of being comprehensive because it has the possibility of covering everyone in the health unit under the responsibility of the community health committee (CHC), according to their need and care need ${ }^{8}$.

The system collects information to help the community health committee plan and manage health activities at the community level, and within parameters set by the Ministry of Health, each community health committee on the scope of the health information system guided by the community 
health extension worker (CHEW); based on their experience and available information from the community, the $\mathrm{CHC}$ prioritizes the problems that determine the indicators to be looked at; possible courses of action are then planned, and the $\mathrm{CHC}$ will identify the type of information to be collected, who collects them and what tools are required, as well as how the information will be analyzed, disseminated, utilized and stored for future use ${ }^{9}$.

The Department for International Development (DFID) in their assessment of how quality of data can be enhanced in Zanzibar noted that data-use workshops with active engagement of data users themselves can improve health information systems overall and enhance staff capacity for information use, presentation and analysis for decision-making ${ }^{4}$.

Essentially, this data collected is based on the activity of the community health workers (CHW), the CHEW and $\mathrm{CHCs}$ as well as general information on the community development issues, socio-economic and demographic indexes of households, community resources, diseases etc. and the type of information collected may include births and deaths, children with child health card, children with complete immunization, number of pregnant women attending antenatal clinics, mothers using oral rehydration salts for diarrhea for their children, use of use of treated nets, latrine coverage, treated drinking water and households with food items in stock etc ${ }^{7}$.

\section{Methodology}

This was a descriptive study design which used qualitative methods of data collection. Sampling was non-probability, convenience sampling from study sites and data collection was done by trained research assistants. Data was collected in Nyalenda, informal settlement (Kisumu) using interview guides for a knowledge, attitude and practice of data use in this location. This data tools was administered by trained research assistants who had to move to health services points in the study area to the study respondents comprising of health managers, health care providers and community health workers; there were 43 community health workers, 4 community health committee chairmen, 4 public health officers, and 15 community health extension workers. This allowed the researchers to understand the practice of the health managers in internalizing the importance of systematic data collection and use in their planning programme.

\section{Data analysis}

Data collected through focus group discussions interviews was subjected to thematic content analysis. The recorded data through Focus Group Discussions was transcribed. Main themes and sub-themes were identified to generate common perceptions and issues which were identified and narratives constructed. Multiple coding was done to create coding categories which were capable of reflecting the content of the data. The coding categories extracted from the transcripts were used to systematically analyze commonalities and apparent perceptions reflected in the data by focusing on issues which were repeatedly mentioned or strongly emphasized by the informants. Responses were compared across the type of respondents (CHWs, Health Service providers and Consumers).

\section{Results}

According to the respondents, CBHIS has been very helpful in providing information which they use in the health facility to improve quality of health care. This data has been used to inform the decisions with regard to health care service delivery to those seeking healthcare from the health facility.

"Through the information it has help us to see the areas which need our services most and through that data we have been able to access those areas and take our services there to assist the community...." (FGD 3)

The CBHIS information has also been very useful in managing conditions like TB whose treatment regimen require high level of adherence; CBHIS has been able to generate information that identifies and helps in tracing drug defaulters. The information has also been useful in areas like antenatal care (ANC) attendance and improving immunization coverage of under-fives.

"Community medical case like a clients on CCC (comprehensive care) to trace may be defaulters, those who have, lost follow up, may be they have transferred and you don't know, we use these information from the community, also in case some people may not be able to come to the clinic we sent some people to trace, so such information do help." (FGD 4)

"Now effectiveness and efficiency of care, this one has come through, it (CBHIS) has led to the introduction of performance appraisals where maybe and also patients chatter, when a patient comes he or she is directed on how to move and also the waiting hours should be reduced, maybe if somebody goes for consultation from there to the lab the time for timing should also be reduced so it's put on every chatter if someone waits for so long he or she should ask for why am I not being served quickly so after that also we create the suggestion box if something does not please the patient he can write a note and put there so after sometimes we sit down and evaluate our performance: lab is not performing, maybe CCC (comprehensive care center) is not performing why there is this complain and this complain we have to improve on this and on financial and social allocation, we have to outline satisfaction first for outlining the satisfaction." (FGD 2)

Information from CBHIS also helps to identify disease outbreak, this occurs when the health managers analyze data from the community, the information is also used to confirm 
the condition and developing control strategies to prevent future occurrence.

"Yes, in case of such a an outbreak usually the data helps us to analyze, it helps us to confirm our diagnosis on condition, it helps us maybe to prevent more such cases to happen (FGD 3)

Generally, this data is used to improve the quality of life in the community and maybe in case of something like outbreaks in the community CHWs report to us and we have gone there maybe to correct the situation, then the quality of life also improves in that we will prevent mortality and morbidity that's maybe improving the quality of life in the community. (KII 1)

When asked on how information they get from the CBHIS has been used to evaluate interventions and how they have used the information to improve program quality and delivery. They indicated that the information has been used to evaluate ANC utilization, immunization coverage and to collect other health indicators like latrine coverage.

"We use the data to plan on how we can improve on immunization and how we can plan for out reaches so that we can be able to cover the areas which are the villages especially the villages with the great number of children who have not completed the immunization... (KII 6)

This information is then analyzed to identify health service utilization gaps; in cases where they find gaps in either utilization of those health services or latrine coverage, health managers always use forum like continued medical education (CME) meetings to bring these to attention of other health worker and managers and they then work together to identify strategies that can be used to improve the programs. Some of the strategies hey suggested during the discussion that they do employ were community education and outreaches, resource allocation and recruitment of more community health workers (CHWs).

"... you know here you have done what, here you have not done what so you may come back to sit down like new normally have monthly CMEs meeting and then it makes us improve on what was not..." (KII 4)

Though CBHIS managers have used the data from CBHIS to improve program implementation and ensure quality of delivery of the intended deliverables; there was a feeling that the aspect of financial commitment from the government has been a great challenge; in most cases the budget is done and approved by the ministry nonetheless activities are never financed.

"We have been planning for the community units' annual budget planning but the money has never come. last year this financial year which has just ended, they only brought the list of item which they were going to fund but the money never came. (FGD 6)

When the respondents were asked on how CBHIS has help in improving the efficiency of management at the health facility, different themes emerged about the efficiency. CBHIS help in conducting management audit to identify where the health workers have performed well and those areas that need improvements and made changes to that effect.

“....for us to improve so it (CBHIS) helps us to perform, where we have not performed in our management. (FGD 9)

Themes also emerged on how CBHIS helps health managers to identify management areas that need to be supported and where urgent actions are needed so as to ensure health service delivery is improved.

....the information has influence the health management to know the areas which need to be supported and where the services are needed most by the use of that data it has help the improve the people who accessing the health services (FGD 3).

“.....CBHIS helps us to do to perform where we have not performed." (KII 5)

Respondents also believed that information from the CBHIS also helps in planning for the emergencies like disease outbreak and other unforeseen health related disasters that might occur and also to plan efficiently for community health education or outreaches.

"Yes they have improved and it assisting because those who are disadvantaged through the information we get from the community now the services are being, the health facility and the management they trying to take their services closure to the community especially they are planning for the out reaches (FGD7)

When we receive outbreak information, it (CBHIS) also helps us through up to the government to plan for future prevention and maybe also alerting the whole region that such a case has happen so preventive measures has to be taken (FGD 8)

There was a different school of thought from other respondents when asked on how CBHIS has helped to improve management efficiency, they felt that though there has been some change in management efficiency due to existence of CBHIS though the change has been very minimal.

The change has been there, so minimal but I can say that there is some little change (KIII)

\section{Discussion}

Through the community health strategy implementation, the gathering and use of information at community level is linked to the Ministry of Health through the health committees (CHC) in the community who essentially regularly meet to assess information collected by the CHWs; these discourse by the CHCs leads to the planning of the dialogue and health action days as a way of responding to 
information about health challenges in the community; this approach is similar to the experience of Braa et al, (2012) in their assessment of how quality of data can be enhanced in Zanzibar; the data-use workshops with active engagement of data users themselves as a unique active way of improving health information systems overall and enhance staff capacity for information use, presentation and analysis for decision-making. In both study sites the planning process were the same and as respondents agreed, this dialogue and health action days have been instrumental in changing the health seeking behavior of the community for better.

Community based health information sharing has contributed to the bridging the interface between community and the health care system through regular dialogue, based on available data. This has improved the performance at the frontlines ${ }^{10}$. The flow of community based information has enhanced the participation of the community in taking care of their own health, as evidenced from the results of this study, and has improved health status. Local people have been found to be capable of executing all the participatory appraisal activities beyond expectations of the external development facilitators ${ }^{11}$.

The system enables the community to follow up on the progress of implementation of planned activities, to determine their successes and constraints in achieving their objectives, their increasing their voice, and access to quality care. The participation of the local people in planning has been found to be effective in that the local people play a meaningful role in information search and planning. This approach is also less expensive in respect of time and money ${ }^{12}$. Less time and money are spent on information gather in grand processing because it is don e by the local people themselves.

The health advantages that accrue to the community can be immeasurable when the process is well managed; respondents observed people being mobilized and sensitized, frequent home visiting by service providers and $\mathrm{CHWs}$, efficient distribution of drugs (dewormers, vitamins and pain killers), help to orphan and vulnerable children and the elderly etc. at the output level and there were direct improvement on health status outcomes such as general community health improving due to lessons learned during community dialogue, more hospital delivery, reduction in malaria since there is increased ITN use etc.

As seen in Zanzibar, information users confessed the importance of information in improving decision making: when the community health workers are well trained, they collect accurate data based on need of the community. There is improvement in referral system at community level which is important. There is need for more frequent community dialogue as it creates the greatest awareness ${ }^{10-11}$.

It is a good thing to use data for dialogue, data reflects the health status of the community, effective, the client can be referred and action taken. Community members come together to agree on objectives that are then tackled.

As in any good system, there are always some unforeseen challenges; in the context of information use, Moneti, (2001), notes that there are challenges in getting reliable information in time from the health management information system, weak capacities of health management information staff, lack of integration among the many parallel data collection systems and inadequate coordination; likewise the experiences of the two study sites demonstrate these facts; the initial challenge already could be seen in ensuring that the CHWs are well trained; CHWs respondents mentioned these frequently as one way of motivating to gain confidence in their work; it is also seen that the sources of information are many and varied which requires continued updates and refresher training so that the CHWs are skilled in gathering these information and collating them. Other management weaknesses such inadequate coordination between the CHW and the CHEW which is reflected in processing referrals, poor linkage, poor information flow; administrative problems of processing reporting tools in time tend to make the exercise hard and response from the facility not up to date.

The main perspectives of various stakeholders included joint ownership of health intervention programs at the community level. The involvement of health workers empowers participants in that the participants are made to be both Learners and facilitators of the development process ${ }^{12}$. In the process, the community become empowered and is able to take charge and ownership of the development processes in their communities. Service consumers, mothers said that they were able to monitor their ANC and the immunization schedule by looking at the $\mathrm{MCH}$ book. By reading it they were able to monitor the baby's growth and this enabled them to manage the child's feeding based on the trend of weight for age, strengthened through dialogue with the CHW at household level. In addition, the service consumers appreciated the platform to voice their concerns, during dialogue days, to prioritize their health issues, and to engage freely with different stakeholders in actions for health improvement. The sources of information most appreciated included the chalk board, maintained by community health workers.

Sohani et al, (2005) demonstrated how groups of local people analyzing their own conditions and choosing their own means of improving them, apply information system as a tool for change. The method is participatory and empowering in that the local people own, analyze, share and use the information while the outsider remains a facilitator ${ }^{13}$. In other words, the local people participate in the processing of the information needed for their development planning. They share, use and own the information ${ }^{13}$. As people participate, they therefore become self - esteemed, confident, motivated and committed to participate in exploring solutions to their challenges. This means that people participate to be empowered to participate ${ }^{12}$.

This means that as they participate, they share and own the outcomes. In this way they become empowered. The best element is that its recognition that indigenous people are capable of articulating their needs in their own way. The main principle of the method is that the facilitator becomes a 
listener, learner and catalyst on equal basis with the subjects. This approach increases participants' self-esteem, confidence, motivation, and commitment to reflect and explore solutions to their needs.

\section{Conclusions}

This study revealed that utilization of data at community level, at the various health systems levels remain an important pillar of community primary health care; the community health workers were rank as key users of these data which brought about important health changes among the people.

Sources of information at the grass root level are many and varied, likewise the subject matters relating to health are many; as community health workers are the main instrument for information gathering, their training is of paramount importance to ensure that accurate data are collected, collated, disseminated and finally used for planning and health interventions in the community.

Coordination of the health information consumers at the community is also of great importance to ensure planning is based on an acceptable and agreed position in which the members own the process of decision making for better outcome.

In sum, this study demonstrated that health managers need an organized information management system to help them plan health care in the community and achieve maximum benefit from their effort support primary care. This can be adequately achieved by working in partnership with the community, government, local and international partners on the ground.

\section{REFERENCES}

[1] Hallvard Lærum, Tom H Karlsen and Arild Faxvaag. Use of and attitudes to a hospital information system by medical secretaries, nurses and physicians deprived of the paper-based medical record: a case report. BMC Medical Informatics and Decision Making. 2004, 4(18) doi:10.1186/1472-6947-4-18
[2] Routine Health Information Network (2003)"The Prism" Workshop paper in Second International RHINO Workshop on enhancing the quality and use of routine information at the district level. Available at: www.cpc.unc.edu/measure/publications/pdf/ws-04-12.pdf Last accessed 8 October 2008.

[3] USAID, Report of a Technical Consultation on Information Systems for Community-Based HIV Programs. 2009

[4] The Department for International Development (DFID) health resource center (2011). Health Management Information Systems. Retrieved from

http://www.eldis.org/healthsystems/hmis/index.htm

[5] Don de Savigny, Harun Kasale, Conrad Mbuya et Graham Reid CRDI, 2008. Fixing health systems.

[6] Afri-Afya, Better Information: Better Health, Influencing grass-root delivery of health services through ICTs, 2001

[7] Government of Kenya, National Development Plan, 2002

[8] George W. Odhiambo-Otieno, Health Management Information Systems in Kenya, Diagnosis and Prescriptions, Moi University Kenya, Unpublished Ph.D. thesis, (2000)

[9] Peter W Gething, Abdisalan M Noor, Catherine A Goodman,Priscilla W Gikandi, Simon I Hay, Shahnaaz K Sharif, Peter M Atkinson, Robert W Snow. Information for decision making from imperfect national data: tracking major changes in health care use in Kenya using geostatistics. BMC Medicine 2007,5(37)

[10] Francesca Moneti. Community-based information systems; 2001

[11] Marlene Roefs and Retha du Plessis. Community-Based Information System (CBIS): Grade 8 learners become researchers and Developers in their own community. 2004

[12] Marlize Booman, Dave N. Durrheim, Kobus La Grange, Carrin Martin, Aaron M. Mabuza, Alpheus Zitha, Frans M. Mbokazi, Colleen Fraser, \& Brian L. Sharp. Using a geographical information system to plan a Malaria control programme in South Africa. 2000

[13] Robert E. Ford, Integrating GIS into e-health initiatives in Africa, 2009

[14] Sohani, S.; Kamau, M.; Alidina, S., Improved health systems through local information management, Aga Khan Health Services (AKHS) (2005) 